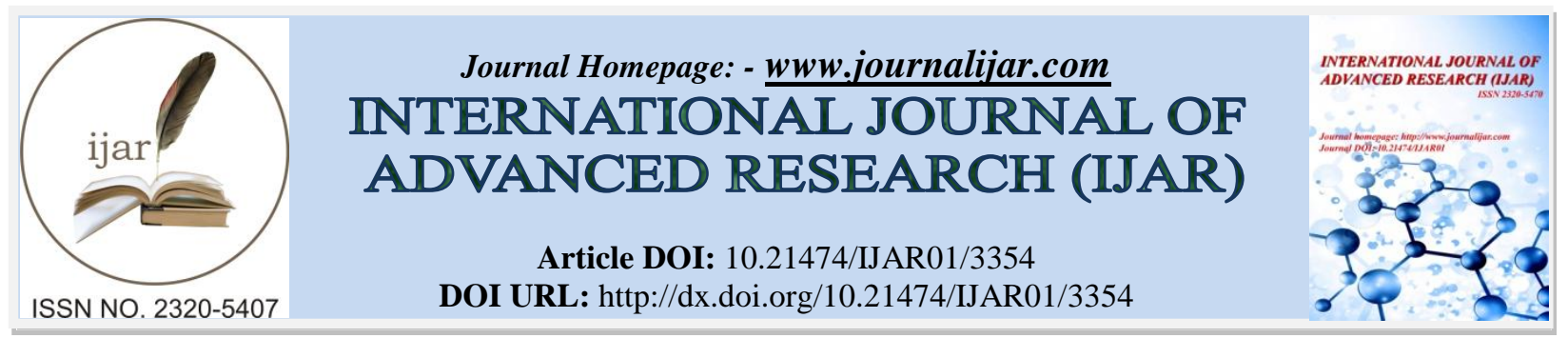

RESEARCH ARTICLE

\title{
REHABILITATION OF PATIENT WITH SQUAMOUS CELL CARCINOMA
}

\author{
Dr. Nazia Majeed Zargar, Dr. Falak Naz and Dr. Jawahir Ahmed Ganai.
}

\section{Manuscript Info}

Manuscript History

Received: 29 December 2016

Final Accepted: 21 January 2017

Published: February 2017

\section{Abstract}

Introduction: Several methods have been advocated for reconstructing maxillectomy defects. The use of an obturator prosthesis is one of them. Effective obturation of maxillary defects produces sufficient separation of the oral and nasal cavity to improve the quality and intelligibility of speech.

Prodecure: A patient aged 65years presented to Government Dental College and Hospital, Srinagar with the chief complaint of swelling in the right posterior region of palate. Patient was completely edentulous in both the arches. Clinical examination revealed a large granular swelling involving the right posterio- lateral portion of hard palate and posterior alveolar ridge. Patient was referred to the Department of Prosthodontics for construction of maxillary obturator. Prosthodontic rehabilitation of the defect was organized into three stages of treatment. Firstly an immediate surgical obturator was fabricated which was placed after the surgical removal of tumour. After 2 weeks impressions were made and an interim obturator was fabricated. After 3 months of healing a patient was given a definitive prosthesis.

Conclusion: Quality of life of patients with maxillary defects can be improved with the provision of a properly designed obturator. The prosthetic obturator can restore mastication, swallowing, esthetic particularly the midface, resonance and speech. Patients with maxillofacial defects who undergo rehabilitation can resume their social habits in the normal way.

Copy Right, IJAR, 2017,. All rights reserved.

\section{Introduction:-}

The maxilla is a functional and esthetic keystone of the mid-face forming part of the mid-facial elements which are the orbit, the zygomatico-maxillary complex, the nasal unit and the stomato-maxillary complex ${ }^{1}$.Maxillofacial defects may be a result of congenital malformations, trauma or surgical resection of tumors. The primary objective of rehabilitating these defects is to eliminate the disease and to improve the quality of life for theseindividuals ${ }^{2}$.Malignant neoplasms of upper gingiva and hard palate nearly account for $1-5 \%$ of total occurrence in the oral cavity; two third of the lesions which involve these areas are squamous cell carcinomas ${ }^{3}$.Unfortunately they spread to adjacent structures, by the time they are diagnosed. The recommended treatment for these type of lesions are alveolectomy, palatectomy, partial/total maxillectomy, depending on the aggressiveness \& location of the actual lesion, its histotype, patient's age and general health status. The post-surgical effect usually has serious consequences as it disturbs both form and function of normal stomatognathic system. Rehabilitation of these acquired maxillary defects can be accomplished by using various type of micro-vascularised flap or by prosthetic 
means. Surgical reconstructions are usually considered when extensions of the defects are small. For larger defect, prosthetic rehabilitation seems to be a better alternative, since more risks are involved for survival of the graft ${ }^{4}$.

Several methods have been advocated for reconstructing these defects. The use of an obturator prosthesis is one of them. Effective obturation of maxillary defects produces sufficient separation of the oral and nasalcavity to improve the quality and intelligibility of speech. It also enhances masticatory function, deglutition and esthetics. Prosthetic intervention should occur at the time of surgical resection and will be necessary for the remainder of the patients $\mathrm{life}^{5,6}$.This present report describes a prosthodontic rehabilitation of a patient with squamous cell carcinoma in the maxilla.

\section{Case Report:-}

A patient aged 65years presented to Government Dental College and Hospital, Srinagar with the chief complaint of swelling in the right posterior region of palate. Patient was completely edentulous in both the arches and presented a history of cigarette smoking from last 35years. Clinical examination revealed a large granular swelling involving the right posterio- lateral portion of hard palate and posterior alveolar ridge (Fig 1). Anincisional biopsy was performed and the sample was sent for histopathological examination which revealed undifferentiated squamous cell carcinoma. Patient was referred to the Department of Prosthodontics for construction of maxillary obturator.

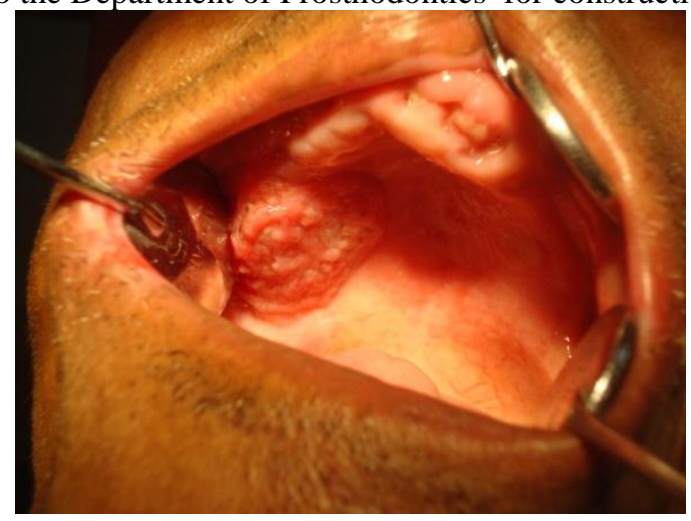

Figure 1:-

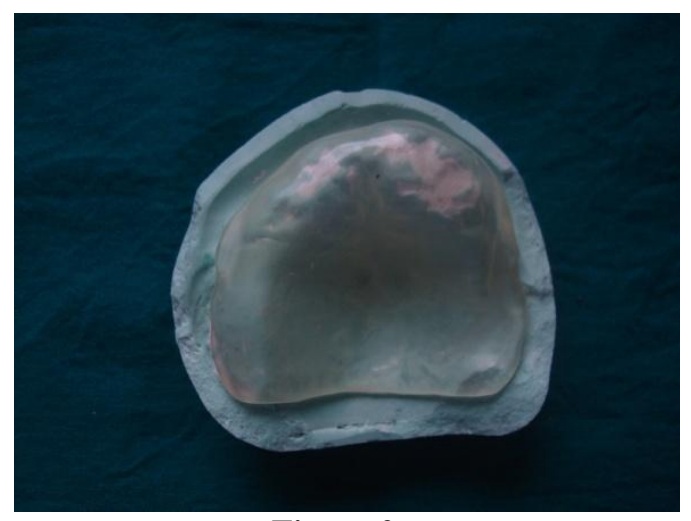

Figure 2:- 


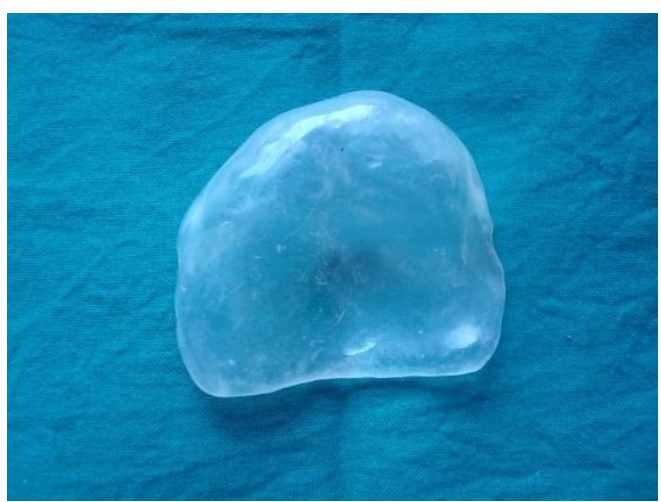

Figure 3:-

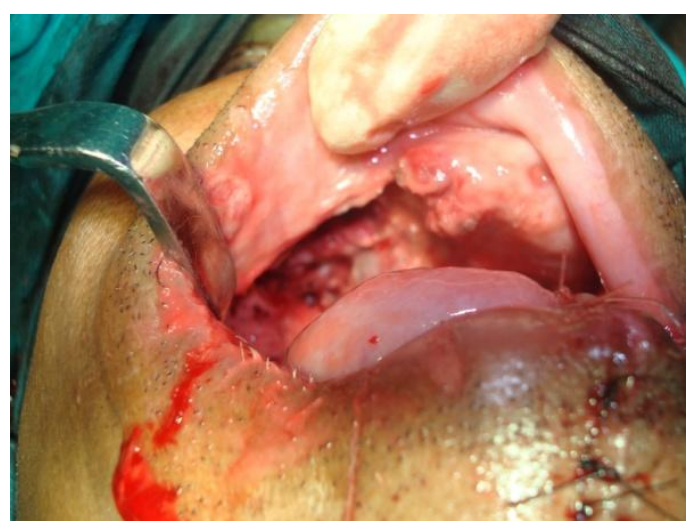

Figure 4:-

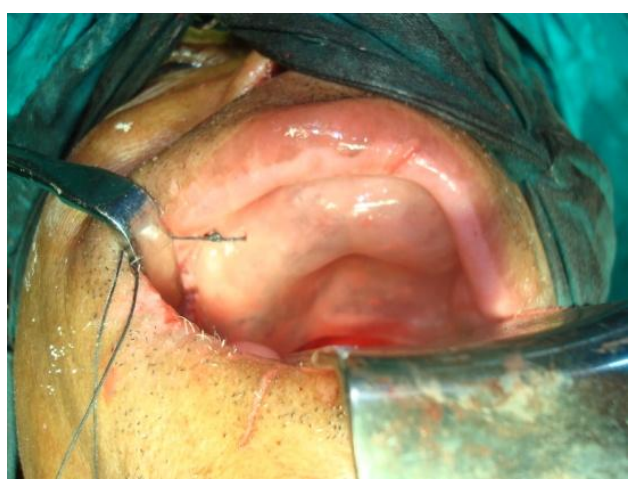

Figure 5:-

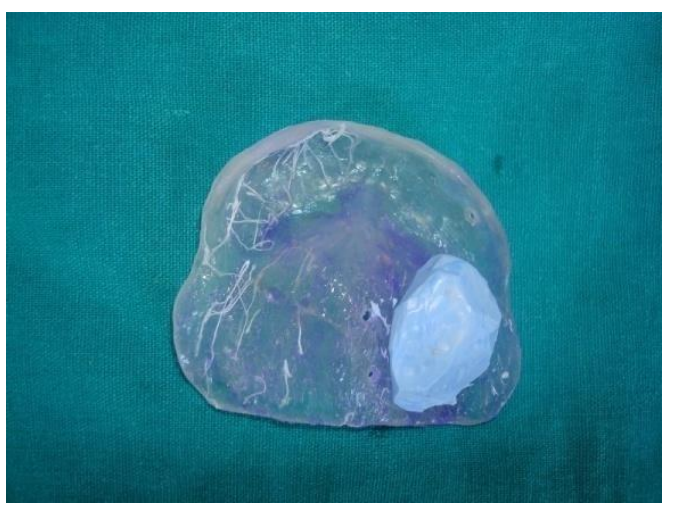

Figure 6:- 


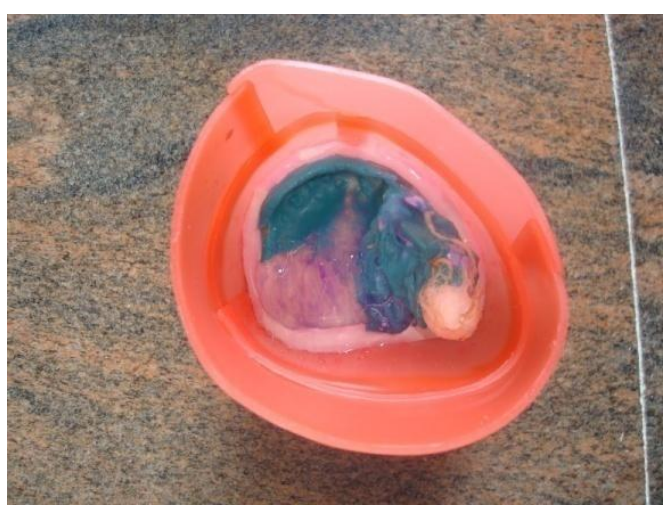

Figure 7:-

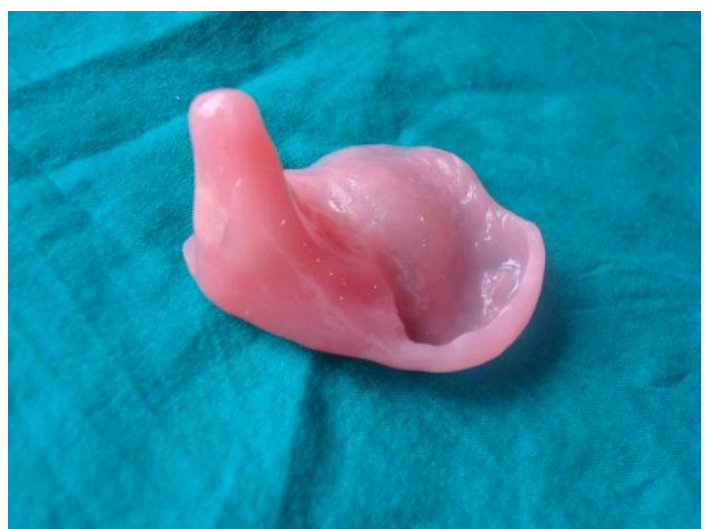

Figure 8:-

\section{Procedure:-}

The pre-operative impression was made using an irreversible hydrocolloid material (Zhermack) in a modified perforated stock tray. The impression was poured with dental stone to produce the cast. The cast was sent to the surgeon to delineate area of resection and an immediate surgical obturator was fabricated using clear auto polymerizing resin (Fig 2,3). In the operating room resection of the maxilla was done to remove the tumor (Fig4) and a surgical closure was attempted using the remaining and neighbouring soft tissues (Fig 5).

The defect was large and the available tissues were not sufficient to completely close the defect. The surgical obturator was inserted immediately after resection of the maxilla and held in position using sutures in the canine fossa region (Fig 5). After two weeks the clinical intra and extraoral examination revealed a good initial healing at the defect site. The extraoral suture was removed at the surgery department.The fabrication of an interim obturator was initialized. The usual steps in fabricating the conventional acrylic partial denture were followed.Surgical obturator served as a custom tray for impression making. Elastomeric impression material was used for recording the details of the defect site (putty polyvinyl silicone followed by a light body wash). Impression was beaded and boxed and poured for fabrication of cast on which an interim obturator was fabricated using heat polymerizing acrylic resin(Fig 6-8).Finally after a period of 3months patient was provided with a definitive prosthesis.

\section{Discussion:-}

Rehabilitation of patients with acquired maxillary defects is relatively simpler than rehabilitation of defects in the mandible, and pleasing as well as accepted outcomes can be identified at the end of treatment. On the other hand, great efforts should be given in dealing with large defects to obtain the substantial requirements for retention and support of theprostheses ${ }^{7}$. Two primary objectives of maxillo-facial prosthodontists in carrying out rehabilitation is to restore the functions of mastication, deglutition, speech and to achieve normal oro-facial appearance ${ }^{8}$.

The aggressive nature and pattern of undifferentiated squamous cell carcinoma in the maxilla makes it difficult to remove completely, which explains the high recurrence rate of this type of tumor. For this reason, rehabilitation with 
prosthetic obturator is preferred. Moreover, the economic factor plays an important role which should be taken into account during treatment decision. Our patient had a low financial income making it difficult for him to meet more advanced treatment modalities.

In this article Prosthodontic rehabilitation of the defect was organized into three stages of treatment. For each step a different type of obturator was fabricated. To start with a simplified version of the obturator in the form of an acrylic plate and gradually converting it to make the intermediate/definite obturator proved to be helpful in relatively faster adaptation of patient to such bigger prosthesis. Quality of life of patients with maxillary defects could obviously be improved with the provision of a properly designed obturator. The prosthetic obturator can restore mastication, swallowing, esthetic particularly the midface, resonance and speech. Patients with maxillofacial defects who undergo rehabilitation can resume their social habits in the normalway'.

\section{Conclusion:-}

Rehabilitation of patients with oral-nasal communication comprises the same steps as those for making conventional prostheses, which does not exclude the need to observe the individuality of each case in the planning and the need to take additional care when taking impressions and installing the appliance. Rehabilitation of such patients provides the tools with which they can resume their daily life such as talking and eating properly.

\section{References:-}

1. Muzaffer AR, Adams WP, Hartog JM, Rohrich RJ, Byrd HS. Maxillary reconstruction; functional and aesthetic considerations. PlastReconstr Surg. 1999;104(7):2172-2183

2. Lethaus B, Lie N, de Beer F, Kessler P, de Baat C, Verdonck HW.Surgical and prosthetic reconsiderations in patients with maxillectomy.J Oral Rehabil. 2010 Feb;37(2):138-42.

3. Tirelli G, Rizzo R, Biasotto M, Di Lenarda R, Argenti B, Gatto A, Bullo F. Obturator prostheses following palatal resection: clinical cases. ActaOtorhinolaryngol Ital. 2010 Feb;30(1):33-39

4. Mukeshsoni,DeshrajJain .Interim Obturator Rehabilitation of a Maxillary Defect -Case Repor.IOSRJDMS.2015;14(2):1-3

5. Taylor T D. Clinical maxillofacial prosthetics. QuintessencePublishing Co,Inc. Pg :85-102.

6. Chalian. Maxillofacial prosthetics.Elsevier publication.

7. BeumerIII J, Marunick MT, Garrett N, Rohner D, Reintsema H,Abemayor E, et al. Rehabilitation of maxillary defects. In: BeumerIII J, Marunick MT, Esposito SJ, editors. Maxillofacial rehabilitation:prosthodontic and surgical management of cancer-related, acquired, and congenital defects of the head and neck. $3^{\text {rd }}$ ed. Chandler Drive: Quintessence; 2011. p. 155-210.

8. Beumer J, Curtis T, Firtell D. Maxillofacial rehabilitation: prosthodontic and surgical considerations. St. Louis: Mosby; 1979. p. 188-243

9. Mohammed Nasser Alhajj,Ibrahim, Nadia Khalifa. Maxillary obturator prosthesis for a hemimaxillectomy patient: A clinical case report.The Saudi Journal for Dental Research 2016: 7; 153-159 